\title{
Eye in the sky
}

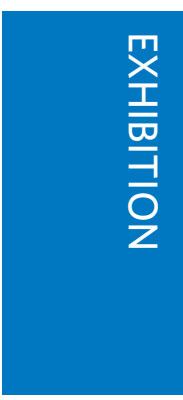

Against the backdrop of increasing excitement and worry about the formidable advances in artificial intelligence (AI) made in the last few years, it is meaningful to review our expectations about this subject through contemporary culture and art, and historical perspectives. This was cleverly achieved by Beyond 2001: Odysseys of Intelligence, an exhibition curated by Claudia Giannetti and hosted by the Espacio Fundación Telefónica in Madrid (31 October 2018-17 February 2019).

The exhibition takes its inspiration from Stanley Kubrick's unforgettable sci-fi movie 2001: A Space Odyssey and deconstructs it into a set of key themes and questions. What does it take to make intelligence morph and evolve - first, together with our biological structures and then, outside of them? This is a kind of general question with which one could approach the exhibition, and possibly no movie could provide a more compelling framework to think about human and artificial intelligence and their relationship.

Indeed, the exhibition takes inspiration from the three-section structure of 2001: A Space Odyssey to guide its own review of the state of the art of AI and its future. The first part concerned the evolution of human intelligence from primates - the ape that 'discovers' how to use a bone as a hammer takes a new path towards a radical reorganization of cognitive and social structures. The exhibition reminds us how, with the subsequent advances in the cognitive and computational sciences over the last century and a half, the evolutionary tree can be juxtaposed to cognitive trees - both those that we find in the brain (represented here by some beautiful century-old drawings of Santiago Ramòn y Cajal; see image), and those that we build in silico. It makes us wonder: are we creating, in the computing machine, a new tool for the next 'leap' of intelligence into new forms of embodiment? The difference between an evolution that mostly happened to us through innumerable small steps in time and that which we are purporting to engineer in electronics should not pass unnoticed. The exhibition, like the movie itself, suggests the case might not be settled smoothly.

The second and largest part of the exhibition maps HAL 9000's capabilities such as vision, learning, planning, search
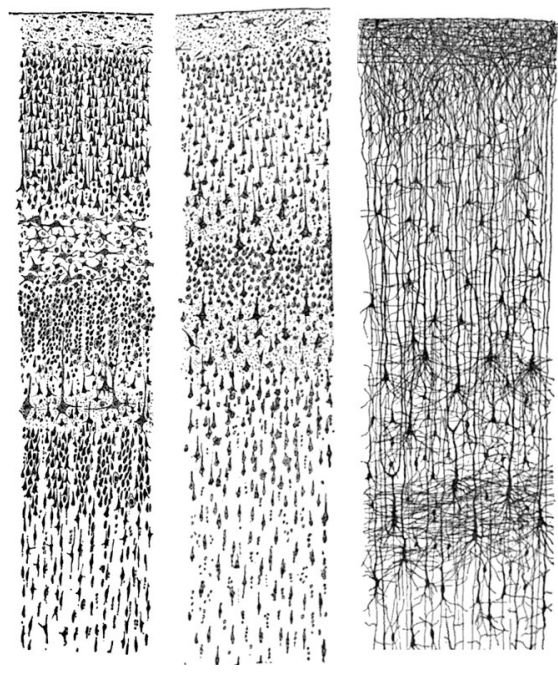

From Ramón y Cajal, S. Comparative Study of the Sensory Areas of the Human Cortex (1899).

and reasoning, and speech and language processing - to today's key research areas in AI. Each capability is the end point of entire programmes of research, and we are reminded about what an exotic machine this 50-year-old imagined computer is still today. How will HAL's 'strong' AI emerge through the eventual integration of these different fields?

This section reviews the state of the art via a set of key questions including: What is AI? Can a machine think? Can AI be creative? Can an AI master human language? Can AI act autonomously? Can an AI system control us? Can AI change our lives? Can AI have emotions? What will human intelligence be for AI? For each of these, what HAL 9000 can do is contrasted to the track record to date.

Take board-game play. HAL 9000 won a chess game against a human in the movie and, less than 50 years later, machines that can play chess and Go have also beaten human intelligence. This completes a long line of attempts that the exhibition tracks back to the automaton developed by Leonardo Torres Quevedo a century earlier in 1912. In another compelling juxtaposition, we can contrast HAL 9000's malfunctions and their damaging consequences with events such as the 'flash crashes' of Western stock markets caused by trading algorithms, here explored by Traders 2018, a multimedia visualization by Daito Manabe, You Tanaka, Kenichiro Shimizy and Shogo Kawata (Goraku). Also, parallels between HAL 9000's ability to visually surveil the astronauts secretly plotting rebellion can be drawn with the advances in surveillance software that can visually recognize and track crowds and individuals (as examined in the documentary Next Level Surveillance, by Paolo Bosonin, Daniel Epstein and Josh Chin, and interactive installation 'Data | ergo sum' by Ana Marcos, Alfonso Villanueva and Iury Lech). Even HAL 9000's artistic sense has been emulated in the real world, and we are shown a machine that can draw a (rather uninspired) portrait of a live subject.

The third section investigates the potential for future AI to become more human-like will computers learn to process emotions? This is crucial if AI will ever fulfil a posthumanist transcendance from its origins in human wetware, but here the distinction between sci-fi literature and real-world prospects seems more blurry. Impressive progress has been made in individual tasks, but we can legitimately wonder about integrating a chessplaying machine with a portrait-drawing one: we would probably get one rather strange kind of disembodied Frankenstein.

How all these deep forays in intelligence emulation will eventually come together is something that the exhibition does not fully tackle. The most interesting point here is not to make a binary prediction about whether we will build higher intelligence, but what are the key points we must evaluate as we do. Interviews with Arthur C. Clarke and Marvin Minsky show they did not have much doubt every inconsistency will be smoothed out. But still, the exhibition leaves us wondering if anyone knows for sure. Not knowing did not obstruct the emergence of our own human intelligence, so for the positively hopeful, this might not be bad news after all.

It might be that no other movie will ever match how Kubrick captured our imagination about the future of intelligence, back in 1968. Fifty years from now, will other productions be remembered for how they opened up questions, expectations and anxieties? For instance, Spike Jonze's Her (2013) is a powerful exploration of AI, and its consequences for the emotional lives of humans; Ex Machina (2014), as well as the series Westworld, investigates themes of meta-consciousness, identity and antagonism in higher embodied $\mathrm{AI}$; and even animation Wall-E, now 10 years old, has invited us to think about artificial emotional intelligence.

Reviewed by Niccolò Tempini ${ }^{1,2}$

${ }^{1}$ University of Exeter, Exeter, UK. ${ }^{2}$ The Alan Turing Institute, London, UK.

e-mail:ntempini@turing.ac.uk

Published online: 1 February 2019 https://doi.org/10.1038/s41567-019-0422-y 